\title{
Improving Clinical Decision Support in Pharmacy: Toward the Perfect DUR Alert
}

\author{
Jenna L. Reynolds, BS, BA, and Michael T. Rupp, PhD
}

\begin{abstract}
SUMMARY
The cornerstone of every health care profession is decision making. Historically, the decisions made by pharmacists have focused on ensuring the accuracy and physical integrity of the pharmaceutical product delivered to the patient in strict compliance with the prescriber's order. As the role of the pharmacist evolved over the past half century, the focus of decision making progressively shifted from a product-centric orientation to optimizing the interaction that occurs between the pharmaceutical product and the patient. Toward that end, prospective drug utilization review (pro-DUR) represents, perhaps, the quintessential expression of the pharmacist's contemporary clinical role.

Fueled by evolving practice standards, innovations in information technology, and legal mandates such as The Omnibus Budget Reconciliation Act of 1990, computer-assisted pro-DUR systems have become a ubiquitous component of pharmacy practice. While these data-driven clinical decision support systems have clearly demonstrated their ability to improve the quality and safety of medication delivery and use, they have yet to fully achieve their promised potential. Doing so will require recognition of continuing shortcomings and a shared commitment by all stakeholders to develop and adhere to best practice guidelines that will better ensure that alerts received by practitioners are valid, interpretable, clinically significant, and actionable.
\end{abstract}

J Manag Care Spec Pharm. 2017;23(1):38-43

Copyright $\odot 2017$, Academy of Managed Care Pharmacy. All rights reserved.

A $\mathrm{t}$ the most fundamental level, the common tie that binds all health care professionals is clinical decision making. In the case of pharmacy, this decision-making role has classically been represented as ensuring that the "five rights" of medication use are achieved: the right drug to the right patient at the right time, dose, and route. ${ }^{1}$

The implementation of the Omnibus Budget Reconciliation Act of 1990 (OBRA '90) codified key aspects of the pharmacist's decision-making role by mandating prospective drug utilization reviews (pro-DUR) to ensure that every medication order is appropriate, medically necessary, and not likely to result in adverse events. ${ }^{2,3}$ Implementation of the DUR provisions of OBRA '90 stimulated a dramatic growth in the development and use of data-driven clinical decision support (CDS) tools in pharmacy. The most prevalent of these were the now-ubiquitous pro-DUR systems that are used by pharmacy computer systems and the prescription benefits management (PBM) industry during prescription claims processing and adjudication. ${ }^{4}$ Properly designed and implemented, such tools can improve clinician decisions and subsequent patient outcomes. ${ }^{5-7}$ However, a growing body of evidence suggests that the full potential of these systems has not been realized in actual practice..$^{8-10}$

Uniform best practice recommendations have yet to be widely adopted to maximize the effectiveness of CDS alerts while minimizing the number of "nuisance alerts" to which users are subjected. ${ }^{11,12}$ The absence of consensus is evidenced by continuing discrepancies among pro-DUR systems in the number, amount, and quality of evidentiary support and clinical relevance of alerts. Usability problems and differences between CDS systems within pharmacy and prescriber systems (e.g., electronic health records [EHRs]) also contribute to the poor acceptance and perceived questionable clinical relevance. ${ }^{13-16}$

There are a number of different types of clinical decision support alerts that are used in medical care, and Troiano et al. (2015) have proposed a hierarchical scheme to distinguish between and among the different types. ${ }^{17}$ Within this framework, the most problematic alerts in pharmacy practice are active, interruptive, database-driven alerts that include drug interactions, dose range and condition, and allergy interaction content.

One of most vexing problems of data-driven pro-DUR systems is alert fatigue. ${ }^{18,19}$ Alert fatigue occurs when systems trigger an unacceptably high number of erroneous, redundant, or clinically irrelevant alerts, thereby becoming a nuisance to users. Ultimately, such nuisance alerts interrupt workflow and distract from patient care, leading to the routine override of alerts by users that have been reported to be as high as $90 \% \cdot{ }^{18,20-22}$

\section{Designing the Perfect DUR Alert}

In the ideal pro-DUR system, every alert would be based on patient-specific and order-specific factors. Theoretical "class effect" alerts would be minimized except where they are severe and relatively likely to occur. The perfect alert would be relevant to, and clinically significant for, the patient and his or her circumstances and well supported by evidence from the clinical literature. ${ }^{23}$ It would be generated at the optimal point in the prescription processing workflow to allow for full consideration and action by the pharmacist without requiring prescriptions to be reworked or claims to be reversed and would be displayed to the pharmacist in a manner that minimizes the possibility of being overlooked or disregarded. 
The perfect DUR alert would be actionable and provide guidance to the pharmacist on the appropriate response, thereby eliminating annoying "FYI alerts." The perfect alert would provide authoritative sources of information, including citations from the clinical literature, that support its generation and would indicate the potential consequences of ignoring the alert. It would also require a documented response that the pharmacist has read and acknowledged the alert and its possible implications.

Successful implementation of any CDS system requires that the "five rights of CDS" are achieved: the right information must be provided to the right person, through the right channel, in the right format, and at the right point in the workflow. ${ }^{24,25}$ And, while there are no quick-and-easy solutions, a number of recommendations may be distilled from the literature to improve the quality, usability, and clinical relevance of pro-DUR systems and related CDS tools, as illustrated in Table 1 and described in the following sections.

\section{The Right Information}

The validity of DUR alerts should be established through an exhaustive, systematic, transparent, and ongoing review of the existing evidence for drug-drug interactions, contraindications, and dosing. Responsibility for ensuring the accuracy and clinical relevance of the information used to generate data-driven DUR alerts ultimately resides with clinical database providers, and significant variability has been found among vendors in the presentation, supporting documentation, supplemental information, and severity or priority of alerts. ${ }^{13,16,26-30}$

Improvements have been made in recent years by the vendors whose databases serve as the source for most pro-DUR alerts. However, the system logic used by pro-DUR programs has not progressed beyond relatively simple rules for alert generation. Recognizing this limitation, calls have been made for improvements in the sophistication of the system logic that is used to generate pro-DUR alerts, including increased use of statistical probability models and branching algorithms to improve accuracy. ${ }^{17}$

Beyond the database providers, pharmacists and health information technology (HIT) systems administrators within health care organizations have a role in ensuring that the right information is provided in alerts. The first step is to identify and suppress unnecessary and unhelpful alerts. Most drug database vendors now allow users to selectively suppress or reclassify alerts. A simple and expedient way to reduce nuisance alerts is to decrease the sensitivity of the alert system to suppress all low-severity alerts. But, while this may seem to be an attractive option, it comes with a serious drawback: an increased likelihood that a potentially important alert will be missed. . $^{31,32}$

A more prudent approach to reducing nuisance alerts is to enlist the assistance of clinical staff to document and report inappropriate or irrelevant alerts and create an expert committee within the organization to review questionable or frequently overridden alerts in order to recommend selective system customizations. ${ }^{32-35}$ When implemented properly, initiatives to selectively reclassify alerts have been successful in eliminating nuisance alerts by as much as $50 \%$, while also increasing user satisfaction. ${ }^{36}$

Reports of overridden high-priority alerts should be run periodically and reviewed by the organization's expert DUR committee or pharmacy management. This retrospective analysis is one way to detect potentially serious alerts that are being overridden by staff and plays an important role in the continuous quality improvement of medication safety management conducted in the organization. ${ }^{37,38}$ Appropriate action should be taken with pharmacy staff that routinely override high-severity alerts without providing an adequate explanation for why the alert was overridden., 40,39

In addition to accuracy, alerts should also be actionable. Vague or ambiguous alerts and those provided for purely informational purposes are seldom helpful in guiding or supporting clinical decisions. To improve actionability, alerts should contain a statement that briefly explains the nature of the alert, how to avoid the danger, and contains a clinically supportable priority indicator (e.g., serious, moderate, minor) with an explanation of the possible consequences of ignoring the alert. ${ }^{40}$

Having the right information to support clinical decisions also implies having adequate information about the medication and the patient for whom it is being prescribed. With respect to the former, the perfect DUR alert begins with ensuring the accuracy of the medication order that is entered into the system. Because transcription errors during medication order entry represent a significant threat to accuracy, the adoption of electronic prescribing (e-prescribing) would seem to be a necessary prerequisite. As for the latter, the contextual information provided by a current medication history is essential if pharmacists are to respond appropriately to alerts of possible threats to medication safety. Pharmacy managers and system vendors can contribute to this by importing pharmacy claims data into the EHR or pharmacy information system. One 2015 study found that combining these 2 sources of medication history resulted in a $17 \%$ improvement in accuracy. ${ }^{41}$

Targeted clinical information such as the patient's diagnosis and/or clinical indication for the medication being prescribed can be extremely helpful in supporting clinical decisions in response to pro-DUR alerts, and the routine transmission of these data are supported by e-prescribing telecommunication standards. ${ }^{42}$ Given the positive effect that this information has demonstrated on improving the quality of pharmacists' DUR decisions, it would seem appropriate to encourage or perhaps even require its routine communication to the pharmacist during the prescribing process. ${ }^{40}$ 


\section{The Right Person}

The best CDS alert is of no value unless it is delivered to the person responsible for making the decision. In the case of pro-DUR, the reviewing pharmacist is ultimately responsible for ensuring that a DUR is conducted on the medication order.

Ensuring that DUR alerts are delivered and displayed to the right person requires support by those responsible for designing pharmacy procedures and practice management systems used in the organization. It should not be possible, for example, for technicians or interns to independently override alerts..$^{14,37}$

Importantly, pharmacists must clearly understand that whether an accurate and appropriate DUR alert reaches them or not, they are the licensed professionals who are ultimately responsible for ensuring that a DUR is performed on each medication order they process and dispense. While such CDS tools as computer-assisted pro-DUR systems can support the clinical judgment of the pharmacist, they are not intended to supplant that judgment. Ultimately, the heavy mantle of responsibility rests exclusively on the pharmacist's shoulders. This is a point that should be continuously reinforced to all staff by the quality and safety managers in the organization.

\section{The Right Channel}

Effectively using the channel through which the DUR alert is delivered requires that users are adequately trained in how to interact with their practice management systems. User training is one of the most important factors in the successful implementation of CDS tools, and inadequate training is one of the most frequently voiced complaints by users. ${ }^{43}$ Unfortunately, it is not uncommon for the pro-DUR "training" that new staff receives from seasoned colleagues in pharmacy organizations to consist primarily of how to quickly bypass system alerts in order to meet organizational productivity expectations. ${ }^{4}$ The perfect DUR alert would be delivered to the pharmacist by a practice management computer system on which the user has been thoroughly trained and assessed to ensure proficiency.

This is an area within which pharmacy educators also have a role to play by preparing students to interact optimally with the data-driven pro-DUR systems. Historically, educators have largely relegated this important aspect of professional training to "on-the-job" training by employers. While pharmacy students receive instruction in the clinical basics of DURs, the plethora of possible pro-DUR systems and platforms that students might find themselves working with after graduation have made formal integration of computer-assisted pro-DUR skills into the professional curriculum seem impractical. That should change. While it is indeed impossible to represent the breadth of systems with which students may find themselves working after graduation, instilling an appreciation for how best to interact with a pro-DUR system will improve their abilities to interface more effectively with this technology and hopefully help them avoid the adoption of bad habits as employees.

\section{The Right Time in Workflow}

To be effective, pro-DUR alerts should be delivered and displayed at the point in the processing and dispensing workflow that is most amendable to taking the desired action. For this reason, the timing of alerts from each pro-DUR application must be customized to the procedures and processes of the organization.

Accomplishing this orchestration requires the collaboration of departments across the organization, including such diverse areas as IT, operations, employee training, and quality/safety management. It is particularly important that relevant contextual information is provided to users concurrently with alerts to ensure that the pharmacist considers this information.

\section{The Right Format}

In their review of medication safety alerts in clinical information systems, Phansalkar et al. (2010) noted that while successfully prompting clinicians at the point of care requires consideration of various factors, "the technological and clinical aspects have received the most attention, while the sociotechnical aspect of alerting, which is related to the human aspect of the interaction between users and technology, has been relatively neglected." ${ }^{\prime 0}$ The authors further note that "successful adoption requires careful consideration of not only the knowledge driving the alerting system but also the human factors principles in alert implementation." ${ }^{40}$

The application of human factors requires that the realistic capabilities and limitations of humans are considered in the design and implementation of CDS systems. Thus, the form the alert takes can be as important as the quality of information it contains. To be effective, CDS alerts must be clearly and prominently displayed to pharmacists in a format that is easily and quickly interpretable and actionable. ${ }^{24}$

Phansalkar et al. suggest considerations that include placement, visibility, color, prioritization, learnability, and confusability when designing and implementing medicationrelated decision support alerts. ${ }^{40}$ These recommendations suggest that to optimize effectiveness, alerts should be easily differentiated by the use of color, shape, and screen position, as well as employing "information only" alerts that do not interrupt workflow and are displayed at an appropriate place on the screen.

In an analysis of drug interaction alerts generated by 14 different EHR systems, Zachariah et al. (2011) noted significant variance in the application of human factors principles, the most common of which included the absence of alert prioritization, clear and concise alert messages, actions for clinical management, and a statement indicating the consequences of overriding an alert. ${ }^{44}$ EHR vendors should design the user interface around human factors principles, a concept that has been neglected in many current systems. ${ }^{44}$ 


\section{TABLE 1 Recommendations to Improve DUR} Alerts in Pharmacy

1. Database vendors and systems administrators should ensure the validity of alerts through a systematic, transparent, and ongoing review of clinical literature to assure users that workflow interruptions are warranted by the potential threat to patient safety that is represented in the alert.

2. The sophistication of system logic that is used to generate pro-DUR alerts should be improved to make increased use of statistical probability models and branching algorithms to improve accuracy.

3. Database vendors and systems administrators should provide users with the flexibility to selectively suppress alerts that are determined to be unnecessary or inappropriate in their practice.

4. Pharmacy organizations should enlist clinical staff to report inappropriate or irrelevant alerts and create an expert committee within the organization to review questionable or frequently overridden alerts for the purpose of recommending system customizations and providing feedback to database providers.

5. DUR alerts should be delivered and integrated at the point in workflow that is most amenable to taking the desired action and should include all requisite contextual patient information to support optimal decision making.

6. Pharmacists must clearly understand that CDS tools such as computerassisted pro-DUR systems are intended to assist and support the clinical judgment of the pharmacist, not supplant that judgment.

7. Pharmacists should be adequately trained to use CDS tools such as computer-assisted pro-DUR, and training should be integrated into the curricula of pharmacy schools rather than being relegated to on-the-job training.

8. Human factor principles should be applied to pro-DUR systems design that consider the importance of color, size, font, and placement of displayed alerts.

9. Displayed alerts should be quickly interpretable and actionable and should include the patient safety consequences of ignoring the alert.

10. Continuous quality improvement of pro-DUR systems should be the shared goal of database and system vendors, systems administrators, pharmacy organizations, and clinicians.

$C D S=$ clinical decision support DUR $=$ drug utilization review

Regardless of system design, it is essential that pharmacists are thoroughly familiar with their alerting systems, since each presents information differently. ${ }^{45}$ Information from the same database vendor or even the same software provider may be presented differently between pharmacies or even within the same pharmacy if customizations have been implemented.

Who, then, is ultimately responsible to ensure that the alert is in the best format and incorporates human factors principles? The database vendors clearly have a responsibility to enable flexibility and customization by users to fine tune alert presentation. However, the customizations that are implemented are ultimately the responsibility of the pharmacy organization and its HIT systems administrators, so a process should be in place to ensure that it is done accurately and in a timely fashion.

\section{Conclusions}

Prospective drug utilization review is a legal, professional, and ethical responsibility of pharmacists. CDS tools can improve
DUR decisions made by pharmacists and, in so doing, improve the safety and effectiveness of medication use by patients. However, the full potential of this technology has yet to be realized, and work remains to be done to achieve the vision of a perfect DUR alerting system. Issues related to what information should be transmitted to pharmacists and other clinicians and how that information should be presented require more attention.

Convening a panel of industry experts would seem to be a worthwhile first step toward the creation of consensus in the design and use of data-driven DUR alert systems. Such a panel should represent the insight and interests of all relevant stakeholders, including, but not limited to, clinical knowledge database suppliers, EHR and practice management computer system vendors, clinicians (pharmacists and prescribers), EHR and e-prescribing software vendor certifying organizations (e.g., Surescripts), HIT standards development organizations (e.g., National Council for Prescription Drug Programs), and representatives from the managed care industry, especially PBMs. The panel should be charged with developing best practice recommendations and initial implementation steps, as well as identifying key unknowns that can serve to direct supportive research efforts. Beyond a national consensus, we believe this initiative should aspire to ultimately create international consensus in the appropriate design and application of CDS tools to improve the safety and effectiveness of medication prescribing and use, since international interoperability considerations are likely to become increasingly important in the future. ${ }^{46}$

\section{Authors}

JENNA L. REYNOLDS, BS, BA, and MICHAEL T. RUPP, PhD, Midwestern University, Glendale, Arizona.

AUTHOR CORRESPONDENCE: Michael T. Rupp, PhD,

Midwestern University, College of Pharmacy, 19555 N. 59th Ave., Glendale, AZ 85308. Tel.: 623.572.3528;

E-mail:mtrupp@midwestern.edu.

\section{DISCLOSURES}

The authors received no funding for this article. Rupp discloses consultancy fees from Surescripts unrelated to this manuscript.

Both authors contributed equally to concept, analysis, and manuscript preparation

\section{REFERENCES}

1. Grissinger M. The five rights: a destination without a map. PT. 2010;35(10):542.

2. U.S. Congress. H.R. 5385, Omnibus Budget Reconciliation Act of 1990. 101st Congress. 1989-1990. Available at: https://www.congress.gov/ bill/10lst-congress/house-bill/5835/text. Accessed December 3, 2016. 
3. National Association of Boards of Pharmacy. Omnibus Budget Reconciliation Act of 1990 patient counseling and drug use review requirements [information packet]. Park Ridge, IL; 1992.

4. Cash JJ. Alert fatigue. Am J Health Syst Pharm. 2009;66(23):2098-101.

5. Kawamoto K, Houlihan C, Balas E, et al. Improving clinical practice using clinical decision support systems: a systematic review of trials to identify features critical to success. BMJ. 2005(7494);330:765.

6. Bates DW, Cohen M, Leape LL, Overhage JM, Shabot MM, Sheridan T. Reducing the frequency of errors in medicine using information technology. J Am Med Inform Assoc. 2001;8(4):299-308.

7. Wolfstadt JI, Gurwitz JH, Field TS, et al. The effect of computerized physician order entry with clinical decision support on the rates of adverse drug events: a systematic review. J Gen Intern Med. 2008;23(4):451-58.

8. Chrischilles E, Fulda TR, Byrns PJ, Winkler SC, Rupp MT, Chui MA. The role of pharmacy computer systems in preventing medication errors. J Am Pharm Assoc (Wash). 2002;42(3):439-48.

9. Chui M, Rupp MT. Evaluation of online prospective DUR programs in community pharmacy practice. J Manag Care Pharm. 2000;6(1):27-32. Available at: http://www.jmcp.org/doi/abs/10.18553/jmcp.2000.6.1.27.

10. Sittig DF, Wright A, Osheroff JA, et al. Grand challenges in clinical decision support. J Biomed Inform. 2008;41(2):387-92.

11. Saleem JJ, Russ AL, Sanderson P, Johnson TR, Zhang J, Sittig DF. Current challenges and opportunities for better integration of human factors research with development of clinical information systems. Yearb Med Inform. 2009;48-58.

12. Roberts LL, Ward MM, Brokel JM, Wakefield DS, Crandall DK, Conlon P. Impact of health information technology on detection of potential adverse drug events at the ordering stage. Am J Health Syst Pharm. 2010;67(2):1838-46.

13. Hines LE, Murphy JE, Grizzle AJ, Malone DC. Critical issues associated with drug-drug interactions: highlights of a multi-stakeholder conference. Am J Health Syst Pharm. 2011;68(10):941-46.

14. Warholak TL, Rupp MT, Zale A, Hines M, Park S. Check it out: a practical tool for improving medication safety. J Am Pharm Assoc (2003). 2015;55(6):621-25.

15. Joint Commission. Safe use of health information technology. Sentinel Alert Event. Issue 54. March 31, 2015. Available at: http://www.jointcommission.org/assets/1/18/SEA_54.pdf. Accessed December 3, 2016.

16. Saverno KR, Hines LE, Warholak TL, et al. Ability of pharmacy clinical decision-support software to alert users about clinically important drugdrug interactions. J Am Med Inform Assoc. 2011;18(1):32-37.

17. Troiano D, Jones MA, Smith AH, et al. ASHP Guidelines on the Design of Database-Driven Clinical Decision Support: strategic directions for drug database and electronic health records vendors. Am J Health Syst Pharm. 2015;72(17):1499-505.

18. Isaac T, Weissman JS, Davis RB, et al. Overrides of medication alerts in ambulatory care. Arch Intern Med. 2009;169(3):305-11.

19. Ash JS, Sittig DF, Campbell EM, Guappone KP, Dykstra RH. Some unintended consequences of clinical decision support systems. AMIA Annu Symp Proc. 2007;26-30. Published online 2007. Available at: https://www.ncbi. nlm.nih.gov/pmc/articles/PMC2813668/. Accessed December 3, 2016.

20. Lee EK, Mejia AF, Senior T, Jose J. Improving patient safety through medical alert management: an automated decision tool to reduce alert fatigue. AMIA Annu Symp Proc. 2010:417-21. Published online November 13, 2010. Available at: https://www.ncbi.nlm.nih.gov/pmc/articles/ PMC3041356/. Accessed December 3, 2016.

21. Weingart SN, Toth M, Sands DZ, Aronson MD, Davis RB, Phillips RS. Physicians' decisions to override computerized drug alerts in primary care. Arch Intern Med. 2003;163(21):2625-31.

22. Van der Sijs H, Aarts J, Vulto A, et al. Overriding of drug safety alerts in computer physician order entry. J Am Med Inform Assoc. 2006;13(2):138-47.
23. Tilson H, Hines LE, McEvoy G, et al. Recommendations for selecting drug-drug interactions for clinical decision support. Am J Health Syst Pharm. 2016;73(8):576-85.

24. Agency for Health Research and Quality. Chapter 1: approaching clinical decision support in medication management. Section 2: overview of CDS five rights. From: Improving medication use and outcomes with clinical decision support: a step-by-step guide. Available at: https://healthit. ahrq.gov/ahrq-funded-projects/clinical-decision-support-initiative/chapter1-approaching-clinical-decision/section-2-overview-cds-five-rights. Accessed December 3, 2016.

25. Campbell R. The five rights of clinical decision support: CDS tools helpful for meeting meaningful use. J AHIMA. 2013;84(10):42-47.

26. Payne TH, Hines LE, Chan RC, et al. Recommendations to improve the usability of drug-drug interaction clinical decision support alerts. J Am Med Inform Assoc. 2015;22(6):1243-50.

27. Abarca J, Malone DC, Armstrong EP, et al. Concordance of severity ratings provided in four drug interaction compendia. J Am Pharm Assoc (2003) 2004;44(2):136-41.

28. Fulda TR, Valuck RJ, Van der Zanden J, et al. Disagreement among drug compendia on inclusion and ratings of drug-drug interactions. Curr Ther Res. 2000;61(8):540-48.

29. Olvey EL, Clauschee S, Malone DC. Comparison of critical drug-drug interaction listings: the Department of Veterans Affairs medical system and standard reference compendia. Clin Pharmacol Ther. 2010;87(1):48-51.

30. Teich JM, Osheroff JA, Pifer EA, Sittig DF, Jenders RA; CDS Expert Review Panel. Clinical decision support in electronic prescribing: recommendations and an action plan: report of the Joint Clinical Decision Support Workgroup. J Am Med Inform Assoc. 2005;12(4):365-76.

31. Van der Sijs H, Aarts J, van Gelder T et al. Turning off frequently overridden drug alerts: limited opportunities for doing it safely. J Am Med Inform Assoc. 2008;15(4):439-48

32. Horn JR, Hansten P. Reducing drug interactions alerts: not so easy. Pharmacy Times. 2007;73(6). Available at: http://www.pharmacytimes.com/ publications/issue/2007/2007-06/2007-06-6593. Accessed December 3, 2016.

33. Shah NR, Seger AC, Seger DL, et al. Improving acceptance of computerized prescribing alerts in ambulatory care. J Am Med Inform Assoc. 2006;13(1):5-11.

34. Horn JR, Hansten PD, Osborn JD, Wareham P, Somani S. Customizing clinical decision support to prevent excessive drug-drug interaction alerts. Am J Health Syst Pharm. 2011;68(8):662-64.

35. Parke C, Santiago E, Zussy B, Klipa D. Reduction of clinical support warnings through recategorization of severity levels. Am J Health Syst Pharm. 2015;72(2):144-48.

36. First DataBank. Case study: MEDITECH hospitals - addressing alert fatigue. Hospital Sisters Health System. 2014. Available at: http://www. fdbhealth.com/ /media/downloads/form\%20required/us/case $\% 20$ study $\% 20$ -\%20fdb\%20alertspace $\% 20-\% 20$ cpoe\%20meditech $\% 20-\% 20$ hospital\%20 sisters.ashx. Accessed December 3, 2016.

37. Institute for Safe Medication Practices. Heed this warning! Don't miss important computer alerts. February 8, 2007. Available at: https://www. ismp.org/newsletters/acutecare/articles/20070208.asp. Accessed December 3, 2016.

38. Cohen MR, Snetzer JL. Do not miss important computer alerts. Hosp Pharm. 2007;42(5):396-99.

39. Beeler PE, Orav EJ, Seger DL, Dykes PC, Bates DW. Provider variation in responses to warnings: do the same providers run stop signs repeatedly? J Am Med Inform Assoc. 2016;23(e1):e93-98.

40. Phansalkar S, Edworthy J, Hellier E, et al. A review of human factors principles for the design and implementation of medication safety alerts in clinical information systems. J Am Med Inform Assoc. 2010;17(5):493-501. 
41. Phansalkar S, Her QL, Tucker AD, et al. Impact of incorporating pharmacy claims data into electronic medication reconciliation. Am J Health Syst Pharm. 2015;72(3):212-17.

42. Dhavle, AA, Rupp MT. Towards creating the perfect electronic prescription. J Am Med Inform Assoc. 2015;22(el):e7-el2.

43. McCullagh LJ, Sofianou A, Kannry J, Mann DM, McGinn TG. User centered clinical decision support tools: adoption across clinician training level. Appl Clin Inform. 2014;5(4):1015-25.

44. Zachariah M, Phansalkar S, Seidling HM, et al. Development and preliminary evidence for the validity of an instrument assessing implementation of human-factors principles in medication-related decision-support systems-I-MeDeSA. J Am Med Inform Assoc. 2011;18(Suppl 1):i62-i72.
45. Institute for Safe Medication Practices. Improving patient safety in community pharmacy: assessing risk and opportunities for change. 2009. Available at: https://www.ismp.org/communityRx/aroc/files/ISMP_AROC. pdf. Accessed December 3, 2016.

46. Cresswell K, Bates DW, Sheikh A. Six ways for governments to get value from health IT. Lancet. 2016;387(10033):2074-75. 